\title{
Impact of exposure to tobacco smoke, arsenic, and phthalates on locally advanced cervical cancer treatment - preliminary results
}

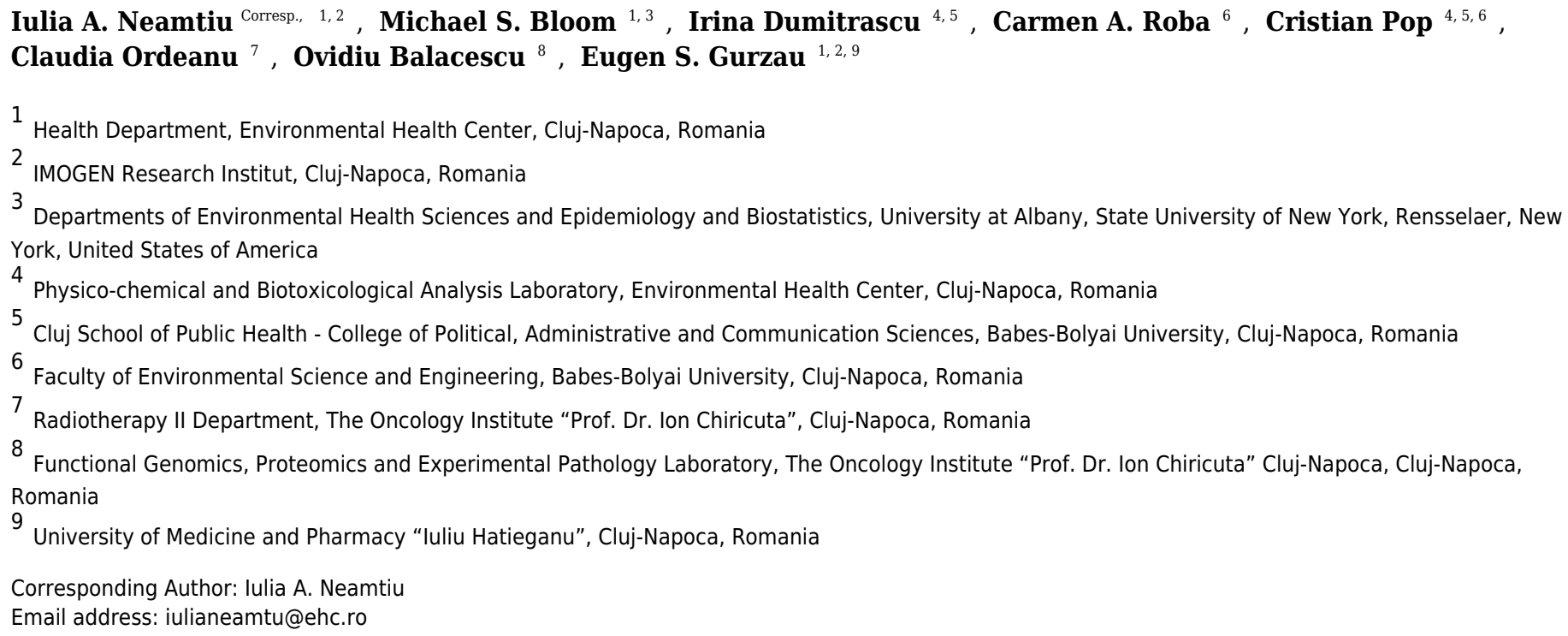

Background. Cancer research is a national and international priority, with the efficiency and effectiveness of current anti-tumor therapies being one of the major challenges with which physicians are faced. Objective. To assess the impact of exposure to tobacco smoke, arsenic, and phthalates on cervical cancer treatment. Methods. We investigated 37 patients with locally advanced cervical carcinoma who underwent chemotherapy and radiotherapy. We determined cotinine and five phthalate metabolites in urine samples collected prior to cancer treatment, by gas chromatography coupled to mass spectrometry, and urinary total arsenic by atomic absorption spectrometry with hydride generation. We used linear regression to evaluate the effects of cotinine, arsenic, and phthalates on the change in tumor size after treatment, adjusted for confounding variables. Results. We detected no significant associations between urinary cotinine, arsenic, or phthalate monoesters on change in tumor size after treatment, adjusted for urine creatinine, age, baseline tumor size, and cotinine (for arsenic and phthalates). However, higher \%mono-ethylhexyl phthalate (\%MEHP), a putative indicator of phthalate diester metabolism, was associated with a larger change in tumor size $(\beta=0.015$, $95 \% \mathrm{Cl}=0.003-0.03, \mathrm{P}=0.019)$. Conclusion. We found no statistically significant association between the urinary levels of arsenic, cotinine, and phthalates metabolites and the response to cervical cancer treatment as measured by the change in tumor size. Still, 
our results suggested that phthalates metabolism may be associated with response to treatment for locally advanced cervical cancer. However, these observations are preliminary and will require confirmation in a larger, more definitive investigation. 
1 Impact of exposure to tobacco smoke, arsenic, and phthalates on locally advanced cervical

2 cancer treatment - preliminary results

3 Iulia A. Neamtiu ${ }^{1,2}$, Michael S. Bloom ${ }^{1,3}$, Irina Dumitrascu ${ }^{4,5}$, Carmen A. Roba ${ }^{6}$, Cristian Pop ${ }^{4,5,6}$,

4 Claudia Ordeanu ${ }^{7}$, Ovidiu Balacescu ${ }^{8}$, Eugen Gurzau ${ }^{1,2,9}$

$5 \quad{ }^{1}$ Health Department, Environmental Health Center, Cluj-Napoca, Romania

$6 \quad{ }^{2}$ Imogen Research Institute Cluj-Napoca, Romania

$7 \quad{ }^{3}$ Departments of Environmental Health Sciences and Epidemiology and Biostatistics,

8 University at Albany, State University of New York, Rensselaer, New York, United States of

9 America

$10{ }^{4}$ Physico-chemical and Biotoxicological Analysis Laboratory, Environmental Health Center,

11 Cluj-Napoca, Romania

$12{ }^{5}$ Cluj School of Public Health - College of Political, Administrative and Communication

13 Sciences, Babes-Bolyai University, Cluj-Napoca, Romania

$14{ }^{6}$ Faculty of Environmental Science and Engineering, Babes-Bolyai University, Cluj-

15 Napoca, Romania

${ }^{7}$ Radiotherapy II, Department, Institute of Oncology “Prof. Dr. Ion Chiricuta”, Cluj-Napoca, Romania

${ }^{8}$ Functional Genomics, Proteomics and Experimental Pathology Laboratory, Institute of Oncology "Prof. Dr. Ion Chiricuta”, Cluj-Napoca, Romania

${ }^{9}$ University of Medicine and Pharmacy “Iuliu Hatieganu”, Cluj-Napoca

21 Corresponding Author: Iulia Neamtiu

58 Busuiocului Street, Cluj-Napoca, postal code 400240, Romania

Email address: $\underline{\text { iulianeamtu@ehc.ro }}$

24

25

\section{Abstract}

Background. Cancer research is a national and international priority, with the efficiency and

effectiveness of current anti-tumor therapies being one of the major challenges with which physicians are faced.

Objective. To assess the impact of exposure to tobacco smoke, arsenic, and phthalates on cervical cancer treatment.

Methods. We investigated 37 patients with locally advanced cervical carcinoma who underwent chemotherapy and radiotherapy. We determined cotinine and five phthalate metabolites in urine samples collected prior to cancer treatment, by gas chromatography coupled to mass spectrometry, and urinary total arsenic by atomic absorption spectrometry with hydride 
34 generation. We used linear regression to evaluate the effects of cotinine, arsenic, and phthalates

35 on the change in tumor size after treatment, adjusted for confounding variables.

36 Results. We detected no significant associations between urinary cotinine, arsenic, or phthalate

37 monoesters on change in tumor size after treatment, adjusted for urine creatinine, age, baseline

38 tumor size, and cotinine (for arsenic and phthalates). However, higher \%mono-ethylhexyl

39 phthalate (\%MEHP), a putative indicator of phthalate diester metabolism, was associated with a

40 larger change in tumor size $(\beta=0.015,95 \% \mathrm{CI}=0.003-0.03, \mathrm{P}=0.019)$.

41 Conclusion. We found no statistically significant association between the urinary levels of

42 arsenic, cotinine, and phthalates metabolites and the response to cervical cancer treatment as

43 measured by the change in tumor size. Still, our results suggested that phthalates metabolism may

44 be associated with response to treatment for locally advanced cervical cancer. However, these

45 observations are preliminary and will require confirmation in a larger, more definitive

46 investigation.

\section{1. Introduction}

48 Cervical cancer is among the leading causes of cancer-related morbidity in women worldwide; in

492012 there were 4,343 new cases in Romania alone, accounting for 1,909 deaths (Ferlay et al.,

50 2013). Response to treatment consisting of radiotherapy combined with cisplatin-based

51 chemotherapy in late stages, in most cases of locally advanced cervical cancers, is good,

52 however, 5-year free survival rates are unsatisfactory (Eifel et al., 2009).

53 Human papilloma virus (HPV) infection (particularly types 16 and 18) is present in more than

$5490 \%$ of cervical cancer cases (Bosch et al., 1995), however, not all HPV infections result in

55 cervical cancer (Burd, 2003). In addition to HPV infection, evidence from epidemiologic studies

56 also supports an association between active cigarette smoking and cervical neoplasia (Haverkos

57 et al., 2003; Trimble et al., 2005). Even in non-smokers, lifetime tobacco exposure may

58 contribute to the development of pre-cancerous cervical intraepithelial neoplasia (Wu et al.,

59 2003). 
60 Exposure to inorganic arsenic has also raised concern worldwide, as it has been associated with

61 lung, bladder, and skin cancer development, among others (Smith et al., 1992). Arsenic induces

62 higher levels of reactive oxygen species (Flora, 2011), an excess of which peroxidizes lipids and

63 oxidizes proteins and nucleic acids, further causing DNA lesions (Ercal, Gurer-Orhan \& Aykin-

64 Burns, 2001). A growing body of evidence indicates that exposure to arsenic may impair the

65 immune system by suppressing $\mathrm{T}$ and $\mathrm{B}$ lymphocyte maturation, inducing apoptosis of

66 macrophages and lymphocytes, and impeding T cell specific cytokine expression (Vega et al.,

67 1999; Cheng et al., 2004), thus promoting cancer development, but arsenic triggered oncogenesis

68 also leads to immune suppression, further facilitating tumor growth and generating a positive

69 feedback loop between cancer and immune function (Acharya et al., 2010), that may also impact

70 the effectiveness of treatment.

71 Phthalates are widely used in many consumer goods, facilitating frequent human exposures

72 (Kamrin, 2009). Phthalates are endocrine disruptors that have been associated with adverse health

73 effects especially as a result of early life exposures (Braun, Sathyanarayana \& Hauser, 2013),

74 exhibiting modulation actions on gonadal steroids that might result in female reproductive health

75 outcomes (Kay, Chambers \& Foster, 2013). Importantly, phthalates also appear to play a role in

76 inflammation, which at a chronic level precedes tumorigenesis (Anand et al., 2008). Phthalates-

77 mediated increases in chronic inflammation have been demonstrated in the prostate, uterus,

78 ovary, and breast, all common locations for neoplastic proliferation (Singh \& Li, 2012).

79 While environmental factors are likely to contribute as component causes in the etiology of

80 cancer (Wu et al., 2016), there appears to be little if any data available to assess effects of

81 environmental pollutants on cancer treatment. Still, it is plausible to speculate that environmental

82 contaminants such as tobacco smoke, arsenic and phthalates, may interfere with cancer treatment.

83 Contaminant-mediated inflammation might modify the immune response to neoplastic 
84 proliferation (Grivennikov, Greten \& Karin, 2010), affecting tumor development and progression,

85 and amplifying (Zitvogel et al., 2008) or decreasing (Ammirante et al., 2010) the response to

86 therapy. Furthermore, these contaminants may impact individual detoxifying capacity and thus,

87 the effectiveness of pharmaceutical agents used for treatment. For example, hepatic cytochrome

88 P450 monooxygenases (CYP450) govern transformation of phthalate diesters to their metabolites

89 (Greenblatt et al., 2002; Frederiksen et al., 2007), the activity of which was decreased in

90 association with exposure to di(2-ethylhexyl) phthalate (DEHP) in a rat model (Parmar,

91 Srivastava \& Seth, 1994). This fact might alter CYP450-dependent metabolic processes for Taxol

92 (one of the chemotherapeutics) (Rowinsky et al., 1993) among DEHP-exposed women. In

93 addition, the investigated contaminants may interact with the chemotherapeutics by competing

94 for binding to carriers/receptors or by producing alteration in tumor tissue or side effects

95 requiring reduction of the therapeutic dose. Both arsenic and total platinum (one of the blood

96 species of the therapeutic agent, carboplatin) bind to proteins (even that protein binding is limited

97 in case of platinum (van der Vijgh, 1991)), and as arsenic binds to protein thiol or sulfhydryl

98 groups, it may act as a competitor for platinum. In fact, clinical trials showed therapeutic value

99 for arsenic trioxide in acute promyelocytic leukemia (Soignet et al., 2001) and myeloma (Deaglio

100 et al., 2001) and few recent studies pointed out that it can produce apoptosis via alterations in

101 specific cell signaling pathways (Seol et al., 1999; Alemany \& Levin, 2000). Finally, response to

102 cervical cancer therapy may differ by its etiology and moreover, the therapy response may differ

103 due in part to environmental contaminants contributing as component causes to cases, including

104 non-HPV confirmed cases, from those attributable solely to HPV infection. As part of a larger

105 investigation into the factors that modify response to treatment among women with invasive

106 cervical cancer, we conducted an interim, exploratory analysis to assess the impact of exposures

107 to tobacco smoke, arsenic and phthalates. To the best of our knowledge, no prior investigations 
108 have assessed the impact of these widely distributed environmental pollutants on the

109 effectiveness of treatment for locally advanced cervical cancer.

110

111

112

113

\section{Materials and Methods}

\section{Study population}

Our study population comprised women receiving treatment for locally advanced cervical cancer at the Oncology Institute "Prof Dr Ion Chiricuta” (Cluj-Napoca, Romania), between 2013 and 2014. Patients were eligible for participation if: 1) diagnosed with histologically-confirmed squamous cell carcinoma of the cervix at clinical stages IIB, IIIA, or IIIB according to the MD Anderson Cancer Center modification of the International Federation of Gynecology and Obstetrics (FIGO) diagnostic criteria (Benedet et al., 2000); 2) aged 20-79 years at the time of diagnosis; 3) diagnosed with Zubrod score $\leq 2$ (an overall well-being index scored as 0-5, for which $0=$ asymptomatic and 5 = death) (Oken et al., 1982); 4) blood hemoglobin $\geq 9$ g/dL, leucocytes $\geq 3000 / \mathrm{mm}^{3}$, and platelets $\geq 100000 / \mathrm{mm}^{3}$; 5) urine creatinine $<1.2 \mathrm{mg} / \mathrm{L}$ and urine nitrogen $<80 \mathrm{mg} / \mathrm{L}$; and 6) normal transaminases. We excluded patients with: 1) a history of a prior malignancy, including previous cervical cancer; 2) interrupted treatment (women who stopped therapy for any reason); or 3) cardiovascular, kidney, or liver function that was deemed too poor to initiate treatment. Of the eligible patients contacted (44 patients), 97.7\% (43 patients) agreed to be enrolled in our study. All participants provided written informed consent prior to study participation and the research protocol was approved by the Ethics Committee in Research and Development and Quality Assurance for Clinical Studies at the Oncology Institute "Prof. Dr. Ion Chiricuta” (approval stated in the Ethics Committee Evaluation Report no. 6490/2013).

All study participants underwent three cycles of chemotherapy with Taxol and Carboplatin (AUC5) followed by radiotherapy (all study participants received the same doses of radiation and 
131 chemotherapeutic drugs), according to the usual clinical cervical cancer treatment protocol, as

132 previously described in detail (Balacescu et al., 2014). We assessed treatment response as the

133 difference in tumor size measured before and after radio and chemotherapy, using a computerized

134 tomography scan. Treatment outcome data were available for 37 (86\%) enrolled participants. Six

135 study participants were still undergoing treatment at the time when the statistical analysis on this

136 preliminary data was done and so outcome data (tumor size after treatment) were not available.

137 Urine samples collection and analysis

138 We analyzed one urine sample, collected prior to cancer treatment, for each study

139 participant. Study nurses collected urine specimens at the time of the cancer diagnosis, into 50

140 mL polyethylene containers previously decontaminated with nitric acid and then rinsed with

141 water. Within 15 minutes of urine collection, samples were frozen at $-20^{\circ} \mathrm{C}$ and then transferred

142 to the Environmental Health Center (Cluj-Napoca, Romania), where they were stored until

143 analysis for cotinine, arsenic, and five phthalate monoesters. Urinary creatinine was measured

144 according to a previously described procedure, (Neamtiu, Dumitrascu \& Roba, 2014), for which

145 the intra-assay coefficient of variation (CV) was 6.5\%.

146 Urinary cotinine - The analytic method for determination of urinary cotinine was

147 previously described in detail (Neamtiu, Dumitrascu \& Roba, 2014). In brief, cotinine was

148 extracted with dichloromethane, dissolved in toluene and analyzed using a QP 2010 Plus NCI gas

149 chromatograph (Shimadzu, Japan) coupled to a mass spectrometer (GC-MS) operated in the

150 selective ion monitoring mode. The method limit of detection (LOD) was $10 \mu \mathrm{g} / \mathrm{L}$ and the intra-

151 assay CV was 3.47\%.

152 Urinary arsenic - The total arsenic concentration in urine samples was analyzed using a

153 Zeenit 700P atomic absorption spectrometer with hydride generation system (Analytik Jena,

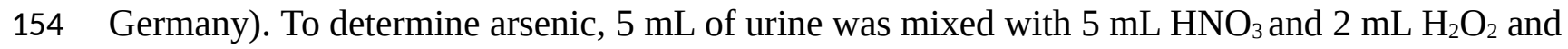


155 mineralized using a Mars 6 microwave digestor (CEM Corporation, USA). The mineralized

156 sample was then diluted to $25 \mathrm{~mL}$ with ultrapure water. The mineralized sample reacts with

157 sodium borohydride in an acid environment and forms volatile metal hydrides, which were

158 atomized in a quartz cell heated at $960^{\circ} \mathrm{C}$. After plotting the calibration curve (arsenic specific

159 wavelength, $\lambda=193.7 \mathrm{~nm})$, the processed samples were atomized and their absorbencies were

160 measured. The method LOD was $0.5 \mu \mathrm{g} / \mathrm{L}$ and the intra-assay CV was $2.36 \%$.

161 Urinary phthalates - We determined urinary phthalate monoester metabolites of dibutyl

162 phthalate (DBP), including mono-butyl phthalate (MBP), benzyl-butyl phthalate (BzBP),

163 including mono-benzyl phthalate (MBzP), and di-ethylhexyl phthalate (DEHP), including mono-

164 (2-ethylhexyl) phthalate (MEHP), mono-(2-ethyl-5-hydroxyhexyl) phthalate (MEHHP), and

165 mono-(2-ethyl-5-oxo-hexyl) phthalate (MEOHP), based on a recently published method (Kim et

166 al., 2014). Briefly, after enzymatic ( $\beta$-glucuronidase) hydrolysis of urine samples, phthalate

167 metabolites were extracted with a solvent mixture (hexane, acetone) by sonication. The organic

168 phase was dried and evaporated to dryness. A derivatization agent, N,O-bis(trimethylsilyl)

169 trifluoroacetamide with trimethylchlorosilane 1\% (BSTFA with TMCS 1\%), was added, and the

170 samples were kept in a thermoreactor (Techne, UK) at $65^{\circ} \mathrm{C}$ for 1 hour. For the analysis, we used

171 a GC-MS QP 2010 Plus NCI (Shimadzu Corporation) in the single ion monitoring mode. The

172 method LOD was $2.5 \mu \mathrm{g} / \mathrm{L}$ and intra-assay CVs were 9.64\% (MBP), 9.01\% (MBzP), 5.43\%

173 (MEHP), 8.7\% (MEOHP), and 4.78\% (MEHHP).

174 Data analysis

175 We characterized distributions for exposures and covariates, and imputed urinary cotinine,

176 arsenic, and phthalates values below the method LODs as LOD $/ \sqrt{ } 2$ prior to analysis (Hornung \&

177 Reed, 1990). We also calculated \%MEHP as [MEHP/(MEHP + MEOHP + MEHHP) x 100] on a

178 molar basis, to assess the impact of phthalates metabolism (Hauser, 2008). To evaluate the 
179 unadjusted effects of environmental exposures on response to cervical cancer therapy, defined as

180 the difference in tumor size before and after treatment, we used a series of individual regression

181 models, including only urine creatinine as a covariate in addition to either cotinine, arsenic, or

182 each phthalate as the sole predictor. We also constructed a series of comprehensive multiple linear

183 regression models to evaluate the impact of confounder adjusted environmental exposures on

184 cervical cancer treatment response, including age, baseline tumor size, and urine cotinine (arsenic

185 and phthalates models) as covariates.

186 Given our use of spot collections for biomarkers of exposure, we adjusted for diurnal variation in

187 urine volume by including creatinine as a covariate in the regression models (Kim et al., 2011).

188 However, in a second set of regression models, we also used a more 'traditional' creatinine

189 correction, in which urinary cotinine, arsenic, and phthalates were divided by urine creatinine and

190 the 'normalized' variables entered into the regression models. We examined the distribution of

191 residuals from all regression models to verify the tenability of the normality assumption and to

192 identify outlying and influential observations for further examination. Stata v.12 (StataCorp LP,

193 College Station, TX USA) was used for the statistical analysis, and statistical significance was

194 defined as $\mathrm{p}<0.05$ for a two-tailed test.

195 3. Results

196 As described in Table 1, 37 participants were 52 years of age on average at the time of the

197 cervical cancer diagnosis (range 26-76 years). Approximately 59\% $(n=22)$ of patients responded

198 well to the cancer treatment, presenting an 80-100\% reduction of the initial tumor size, although

199 the tumor size was reduced by less than $80 \%$, in $41 \%$ of women. Most participants (59\%) were

200 non-smokers (including 13 women who reported tobacco exposure), 6 were former smokers

201 (16\%), and 9 (24\%) women self-identified as smokers.

202 Table 2 shows the distributions for measured urinary cotinine, arsenic, and phthalates levels on a 
203

204

205

206

207

208

209

210

211

212

213

214

215

216

217

218

219

220

221

222

223

224

225

226

227

creatinine basis. Values are reported on a wet-weight basis in the Supplementary Material (Table

S1). Urinary cotinine values ranged from < LOD-395.4 $\mu \mathrm{g} / \mathrm{g}$ creatinine with a geometric mean of

$13.9 \mu \mathrm{g} / \mathrm{g}$ creatinine. Total arsenic values ranged from < LOD-115.4 $\mu \mathrm{g} / \mathrm{g}$ creatinine with a

geometric mean of $13.1 \mu \mathrm{g} / \mathrm{g}$ creatinine. Using ANOVA, cotinine was higher in smokers

compared to non-smokers $(\mathrm{p}=0.04)$ and to former smokers $(\mathrm{p}=0.08)$. On a creatinine basis,

geometric mean values for 35 women with sufficient urine volume available for phthalates

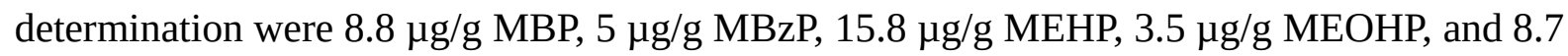

$\mu \mathrm{g} / \mathrm{g}$ MEHHP. The highest maximum values were measured for MBP (295.5 $\mu \mathrm{g} / \mathrm{g})$ and MBzP

(182.9 $\mu \mathrm{g} / \mathrm{g})$, whereas the other phthalates metabolites had lower maximum levels: MEHP (91.9

$\mu \mathrm{g} / \mathrm{g})$, MEOHP (34.1 $\mu \mathrm{g} / \mathrm{g})$, and MEHHP (88.6 $\mu \mathrm{g} / \mathrm{g})$. The geometric mean \%MEHP was 68.2,

ranging from a minimum value of $10.7 \%$ to a maximum value of $97.2 \%$.

Table 3 describes the multiple linear regression analysis of cervical cancer treatment response, measured as the change in tumor size, upon environmental exposures, adjusted for confounding variables. All effect estimates were of small magnitude and 95\% confidence intervals (95\%CI) included the null hypothesis; we detected no statistically significant confounder-adjusted associations between urinary cotinine, total arsenic, or phthalate metabolites and the change in tumor size. We repeated the model without adjusting for cotinine, with no meaningful change in results (data not shown). In contrast, the results for the unadjusted regression analysis of total arsenic were statistically significant $(\beta=0.01,95 \%$ CI $0.0003,0.02 ; \mathrm{P}=0.045)$, as was an age-only adjusted regression analysis $(\beta=0.012,95 \%$ CI 0.002, 0.023; $\mathrm{P}=0.025)$, although results were similarly null in the fully adjusted model. The results were also similar when we used a traditional creatinine correction procedure in lieu of adjustment for urinary creatinine as a covariate in regression models (Supplementary Material Table S2). However, as described by Figure 1 we detected a statistically significant positive association between \%MEHP and response to cervical cancer therapy, in the multivariable regression model adjusted for 
228

229

230

231

232

233

234

235

236

237

238

239

240

241

242

243

244

245

246

247

248

249

250

251

252

confounders ( $\beta=0.015$; 95\% CI 0.003, 0.03; $\mathrm{P}=0.019$ ). However, \%MEHP results were not statistically significant in an unadjusted model $(\beta=0.004 ; 95 \%$ CI $-0.01,0.02 ; \mathrm{P}=0.634)$ or adjusted for age only ( $\beta=0.012 ; 95 \%$ CI $-0.007,0.031 ; \mathrm{P}=0.205)$.

\section{Discussion}

We evaluated the impact of exposure to tobacco smoke, arsenic, and phthalates on locally advanced cervical cancer treatment using urine biomarkers from 37 women aged 26-76 years.

More than a half of the study participants responded well to cancer treatment, presenting a reduction of the initial tumor size between $80 \%$ and $100 \%$. We found no meaningful effects on the change in tumor size for urine cotinine or total arsenic, or for five monoester metabolites of three widely distributed phthalate diesters. Yet, our analysis of \%MEHP suggested an enhanced response to cervical cancer treatment, indicating that less efficient conversion of the primary hydrolytic DEHP phthalate monoester MEHP, to its secondary-oxidative metabolites MEOHP and MEHHP, may confer benefit (Hauser, 2008). It is tempting to speculate the existence of a common metabolic pathway impacting hydrolysis and oxidation of DEHP and response to the cervical cancer treatment protocol. Contaminant-associated modulation of normal metabolic processes (e.g., phthalates) is also likely to impact the pharmacokinetics of chemotherapeutic drugs (Greenblatt et al., 2002). Reduced drug metabolism might result in a longer half-life of the drug, increasing the potential for dose related adverse health effects. In contrast, accelerated drug metabolism might result in a shorter half-life of the drug, reducing its effectiveness. However, given the limited sample size available for our study, and the absence of more comprehensive indicators to characterize cervical cancer therapy response, these observations should be considered preliminary and will require confirmation in a more definitive investigation. Several human biomonitoring studies from nearby European areas reported urinary levels of cotinine, arsenic, and phthalates measured in general population samples. Geometric mean urinary cotinine was modestly lower in 120 Romanian mothers 25 to 45 years of age $(9.10 \mu \mathrm{g} / \mathrm{g}$ 
253 creatinine) (Lupsa et al., 2015) than for women in our study. However, the geometric mean total

254 urine arsenic value for 4,730 Germans aged 18 to $69(3.1 \mu \mathrm{g} / \mathrm{g})$ was substantially lower than for 255 women in our study (Becker et al., 2003). In our study, we determined urinary metabolites of

256 DBP, BzBP, and DEHP as those phthalates most prevalent in Central and Eastern European

257 populations (Černá et al., 2015). The highest urinary phthalates values in our study population

258 were measured for MBP, MBzP, MEHP and MEHHP, while MEHP had the highest geometric

259 mean. The geometric mean concentrations of MBzP and MEHP were higher in our study than

260 were reported for 117 women from the Czech Republic (4.35 and $3.13 \mu \mathrm{g} / \mathrm{g}$ creatinine,

261 respectively), 125 women from Slovakia (3.81 and $3.22 \mu \mathrm{g} / \mathrm{g}$ creatinine, respectively), and 115

262 women from Hungary (3.82 and $3.70 \mu \mathrm{g} / \mathrm{g}$ creatinine, respectively) (Černá et al., 2015). In

263 contrast, levels of MEOHP and MEHHP were lower in our study, than reported for Czech (11.68

264 and $18.45 \mu \mathrm{g} / \mathrm{g}$ creatinine, respectively), Slovakian (11.54 and $18.20 \mu \mathrm{g} / \mathrm{g}$ creatinine,

265 respectively), and Hungarian (10.37 and 15.54 g/g creatinine, respectively) women (Černá et al.,

266 2015). Overall, the distribution of exposures among our study population was unique, yet we

267 identified similarities with values reported for several nearby, non-clinical European populations.

268 Our study had several important limitations, and so, our results should be interpreted only as

269 hypothesis generating. The small number of study participants may have limited our ability to

270 detect modest associations, and it precluded an analysis of multiple exposures included in a single

271 regression model. We hope to expand the sample size in the future to generate more precise effect

272 estimates and to more comprehensively analyze exposure to the mixture of tobacco smoke,

273 arsenic, and phthalates. While our study was prospective in nature, we measured exposure at only

274 a single time point prior to initiation of cancer treatment; the short in vivo $1 / 2$-lives and the

275 episodic nature of phthalates exposure may have misclassified some patients (Fromme et al.,

276 2007). Our use of a total arsenic variable, including comparatively innocuous organic species

277 with toxic inorganic species, may have further misclassified exposure for some women 
278 (Marchiset-Ferlay, Savanovitch \& Sauvant-Rochat, 2012). Yet, misclassification is unlikely to

279 have differed by study outcome and so any bias will have led to underestimated effects. Finally,

280 we did not incorporate recent data suggesting an important role for gene expression on cancer

281 treatment response in our study population (Balacescu et al., 2014). Still, we do anticipate a link

282 to exposure and so bias was unlikely.

283 To the best of our knowledge, this preliminary report describes the first investigation on the

284 impact of widely distributed environmental contaminants on the effectiveness of locally advanced

285 cervical cancer therapy. These results should help to reassure clinicians that even levels of

286 cotinine, total arsenic, MBzP, and MEHP higher than reported from other European study

287 populations are unlikely to interfere with the effectiveness of radio and chemotherapy for

288 invasive cervical cancer. Still, \%MEHP may prove important. Susceptibility to therapy might still

289 differ substantially by exposure status and by HPV status in patients diagnosed with advanced

290 cervical cancer, so we plan to stratify the analysis by HPV status in an expanded future study

291 sample. In this particular case, a linear regression overall probably would not show any strong

292 effects, but among a small group of patients the exposure status might still be a valuable predictor

293 of therapy effectiveness.

294 In a larger future investigation we will incorporate longitudinal collection of urine specimens

295 during cancer treatment to reduce exposure misclassification as well as gene expression

296 information to assess the impact on treatment response. We also plan to evaluate the impact of

297 environmental contaminants (cotinine, arsenic and phthalates) on post transcriptional targets

298 (micro RNA (miRNA)), in the context of HPV-type, to delineate prognostic indicators for

299 treatment effectiveness of advanced stage (IIB-IIIB) cervical cancer.

300 Current cancer treatments are both expensive and induce serious side effects, and so

301 characterizing the potential impact of widely distributed environmental pollutants is critical to

302 establish new indicators for predicting the effectiveness of cervical cancer treatments. 
303

304

305

306

307

308

309

310 311 not have been possible.

\section{Conclusions}

\section{Acknowledgement}

Exposure to tobacco smoke, arsenic, and phthalates did not appear to impact cervical cancer treatment at the levels of exposure experienced by our study population. Phthalates metabolism may be associated with locally advanced cervical cancer treatment response, although the clinical relevance is unclear. A more comprehensive investigation with a larger sample size will be necessary for a more definitive result.

The authors would like to thank the study participants, without whom this investigation would 
313

314

Acharya S, Chaudhuri S, Chatterjee S, Kumar P, Begum Z, Dasgupta S, Flora SJS, Chaudhuri S. 2010. Immunological Profile of Arsenic Toxicity: A Hint Towards Arsenic-induced Carcinogenesis. Asian Pacific Journal of Cancer Prevention 11(2):479-490.

Alemany M, Levin J. 2000. The effects of arsenic trioxide on human Megakaryocytic leukemia cell lines with a comparison of its effects on other cell lineages. Leukemia Lymphoma 38(12):153-163.

Ammirante M, Luo JL, Grivennikov S, Nedospasov S, Karin M. 2010. B cell-derived lymphotoxin promotes castration-resistant prostate cancer. Nature 464(7286):302-305. DOI: 10.1038/nature08782.

Anand P, Kunnumakara AB, Sundaram C, Harikumar KB, Tharakan ST, Lai OS, Sung B, Aggarwal BB. 2008. Cancer is a preventable disease that requires major lifestyle changes. Pharmaceutical Research 25(9):2097-2116. DOI: 10.1007/s11095-008-9661-9.

Balacescu O, Balacescu L, Tudoran O, Todor N, Rus M, Buiga R, Susman S, Fetica B, Pop L, Maja L, Visan S, Ordeanu C, Berindan-Neagoe I, Nagy V. 2014. Gene expression profiling reveals activation of the FA/BRCA pathway in advanced squamous cervical cancer with intrinsic resistance and therapy failure. BMC Cancer 14:246. DOI:10.1186/1471-2407-14246.

Becker K, Schulz C, Kaus S, Seiwert M, Seifert B. 2003. German Environmental Survey 1998 (GerES III): environmental pollutants in the urine of the German population. International Journal of Hygiene and Environmental Health 206(1):15-24. DOI: http://dx.doi.org/10.1078/1438-4639-00188.

Benedet JL, Bender H, Jones H, Ngan HYS, Pecorelli S. 2000. Oncology FCG: FIGO staging classifications and clinical practice guidelines in the management of gynecologic cancers. International Journal of Gynecology and Obstetrics 70(2):209-262.

Bosch FX, Manos MM, Munoz N, Sherman M, Jansen AM, Peto J, Schiffman MH, Moreno V, Kurman R, Shah KV. 1995. Prevalence of human papilloma virus in cervical-cancer - a worldwide perspective. Journal of the National Cancer Institute 87(11):796-802. DOI: 10.1093/jnci/87.11.796.

Braun JM, Sathyanarayana S, Hauser R. 2013. Phthalate exposure and children's health. Current Opinion in Pediatrics 25(2):247-254. DOI: 10.1097/MOP.0b013e32835e1eb6.

Burd EM. 2003. Human papillomavirus and cervical cancer. Clinical Microbiology Reviews 16(1):1-17. DOI: 10.1128/CMR.16.1.1-17.2003.

Černá M, Malý M, Rudnai P, Középesy S, Náray M, Halzlová K, Jajcaj M, Grafnetterová A, Krsková A, Antošová D, Forysová K, Den Hond E, Schoeters G, Joas R, Casteleyn L, Joas A, Biot P, Aerts D, Angerer J, Bloemen L, Castaño A, Esteban M, Koch HM, KolossaGehring M, Gutleb AC, Pavloušková J, Vrbík K. 2015. Case study: Possible differences in phthalates exposure among the Czech, Hungarian, and Slovak populations identified based on the DEMOCOPHES pilot study results. Environmental Research 141:118-124. DOI: 10.1016/j.envres.2014.10.025.

Cheng HY, Li PA, David M, Smithgall TE, Feng L, Lieberman MW. 2004. Arsenic inhibition of the JAK-STAT pathway. Oncogene 23 (20):3603-12 
354

355

356

357

358

359

360

361

362

363

364

365

366

367

368

369

370

371

372

373

374

375

376

377

378

379

380

381

382

383

384

385

386

387

388

389

390

391

392

393

394

395

396

397
Deaglio S, Canella D, Baj G, Arnulfo A, Waxman S, Malavasi F. 2001. Evidence of an immunologic mechanism behind the therapeutic effects of arsenic trioxide on myeloma cells. Leukemia Research 25(3):227-235.

Eifel PJ, Jhingran A, Levenback CF, Tucker S. 2009. Predictive value of a proposed subclassification of stages I and II cervical cancer based on clinical tumor diameter. International Journal of Gynecological Cancer 19(1):2-7. DOI: 10.1111/IGC.0b013e318197f185.

Ercal N, Gurer-Orhan H, Aykin-Burns N. 2001.Toxic metals and oxidative stress part I: mechanisms involved in metal-induced oxidative damage. Current Medicinal Chemistry 1(6):529-539. DOI: 10.2174/1568026013394831.

Ferlay J, Steliarova-Foucher E, Lortet-Tieulent J, Rosso S, Coebergh JWW, Comber H, Forman D, Bray F. 2013. Cancer incidence and mortality patterns in Europe: Estimates for 40 countries in 2012. European Journal of Cancer 49(6):1374-1403. DOI: 10.1016/j.ejca.2012.12.027.

Flora SJS. 2011. Arsenic-induced oxidative stress and its reversibility. Free Radical Biology and Medicine 51(2):257-281. DOI:10.1016/j.freeradbiomed.2011.04.008.

Frederiksen H, Skakkebaek NE, Andersson AM. 2007. Metabolism of phthalates in humans. Molecular Nutrition and Food Research 51(7):899-911. DOI: 10.1002/mnfr.200600243.

Fromme H, Bolte G, Koch HM, Angerer J, Boehmer S, Drexler H, Mayer R, Liebl B. 2007. Occurrence and daily variation of phthalate metabolites in the urine of an adult population. International Journal of Hygiene and Environmental Health 210(1):21-33.

Greenblatt DJ, von Moltke LL, Harmatz JS, Shader RI. 2002. Pharmacokinetics, pharmacodynamics, and drug disposition. In: Davis KL, Charney D, Coyle JT, Nemeroff C, eds. Neuropsychopharmacology: The Fifth Generation of Progress. Philadelphia, Pennsylvania: Lippincott, Williams \& Wilkins, 507-524.

Grivennikov SI, Greten FR, Karin M. 2010. Immunity, inflammation, and cancer. Cell 140(6): 883-899. DOI: 10.1016/j.cell.2010.01.025.

Hauser R. 2008. Urinary phthalate metabolites and semen quality: A review of a potential biomarker of susceptibility. International Journal of Andrology 31(2):112-116. DOI: 10.1111/j.1365-2605.2007.00844.x.

Haverkos HW, Soon GX, Steckley SL, Pickworth W. 2003. Cigarette smoking and cervical cancer: Part I: a meta-analysis. Biomedicine \& Pharmacotherapy 57(2):67-77.

Hornung RW, Reed LD. 1990. Estimation of average concentration in the presence of nondetectable values. Applied Occupational and Environmental Hygiene 5(1):46-51. DOI: 10.1080/1047322X.1990.10389587.

Kamrin MA. 2009. Phthalate risks, phthalate regulation, and public health: a review. Journal of Toxicology and Environmental Health Part B: Critical Reviews 12(2):157-174. DOI: 10.1080/10937400902729226.

Kay VR, Chambers C, Foster WG. 2013. Reproductive and developmental effects of phthalate diesters in females. Critical Reviews in Toxicology 43(3):200-219. DOI: 10.3109/10408444.2013.766149.

Kim K, Steuerwald AJ, Parsons PJ, Fujimoto VY, Browne RW, Bloom MS. 2011. Biomonitoring for exposure to multiple trace elements via analysis of urine from participants in the Study of Metals and Assisted Reproductive Technologies (SMART). Journal of Environmental 
398

399

400

401

402

403

404

405

406

407

408

409

410

411

412

413

414

415

416

417

418

419

420

421

422

423

424

425

426

427

428

429

430

431

432

433

434

435

436

437

438

439

440

441

Monitoring 13(9):2413-2419. DOI: 10.1039/c1em10341e.

Kim M, Song NR, Choi J-H, Lee J, Pyo H. 2014. Simultaneous analysis of urinary phthalate metabolites of residents in Korea using isotope dilution gas chromatography-mass spectrometry. Science of the Total Environment 470:1408-1413. DOI:

10.1016/j.scitotenv.2013.07.037.

Lupsa I-R, Nunes B, Ligocka D, Elena Gurzau A, Jakubowski M, Casteleyn L, Aerts D, Biot P, Den Hond E, Castaño A, Esteban M, Kolossa-Gehring M, Fiddicke U, Knudsen L E, Schoeters G, Reis FM. 2015. Urinary cotinine levels and environmental tobacco smoke in mothers and children of Romania, Portugal and Poland within the European human biomonitoring pilot study. Environmental Research 141:106-117. DOI: 10.1016/j.envres.2015.03.018.

Marchiset-Ferlay N, Savanovitch C, Sauvant-Rochat MP. 2012. What is the best biomarker to assess arsenic exposure via drinking water? Environ Int 39 (1):150-171.

Neamtiu IA, Dumitrascu I, Roba C. 2014. Chemical analysis of urinary cotinine in subjects exposed to a mixture of contaminants. Indian Journal of Applied Research 4(3):56-58. DOI: 10.15373/2249555X/March2014/18.

Oken MM, Creech RH, Tormey DC, Horton J, Davis TE, McFadden ET, Carbone PP. 1982. Toxicity and response criteria of the Eastern Cooperative Oncology Group. American Journal of Clinical Oncology 5(6):649-656. DOI: 10.1097/00000421-198212000-00014.

Parmar D, Srivastava SP, Seth PK. 1994. Age related effects of di(2-ethylhexyl)phthalate on hepatic cytochrome P450 monooxygenases in Wistar rats. Pharmacology and Toxicology 75(3-4):177-180. DOI: 10.1111/j.1600-0773.1994.tb00343.x.

Rowinsky EK, Wright M, Monsarrat B, Lesser GJ, Donehower RC. 1993. Taxol: pharmacology, metabolism and clinical implications. Journal of Cancer Survivorship 17:283-304.

Seol JG, Park WH, Kim ES, Jung CW, Hyun JM, Kim BK, Lee YY. 1999. Effect of arsenic trioxide on cell cycle arrest in head and neck cancer cell-line PCI-1. Biochemical and Biophysical Research Communications 265(2):400-404.

Singh S, Li SS-L. 2012. Bisphenol A and phthalates exhibit similar toxicogenomics and health effects. Gene 494(1):85-9. DOI: 10.1016/j.gene.2011.11.035.

Smith AH, Hopenhayn-Rich C, Goeden HM, Hertz-Picciotto I, Duggan HM, Wood R, Kosnett MJ, Smith MT. 1992. Cancer risks from arsenic in drinking water. Environmental Health Perspectives 97:259-267.

Soignet SL, Frankel SR, Douer D, Tallman MS, Kantarjian H, Calleja E, Stone RM, Kalaycio M, Scheinberg DA, Steinherz P, Sievers EL, Coutré S, Dahlberg S, Ellison R, Warrell RP Jr. 2001. United States multicenter study of arsenic trioxide in relapsed acute promyelocytic leukemia. Journal of Clinical Oncology 19(18):3852-3860.

Trimble CL, Genkinger JM, Burke AE, Hoffman SC, Helzlsouer KJ, Diener-West M, Comstock GW, Alberg AJ. 2005. Active and passive cigarette smoking and the risk of cervical neoplasia. Obstetrics \& Gynecology 105(1):174-18. DOI: 10.1097/01.AOG.0000148268.43584.03.

Van der Vijgh WJ. 1991. Clinical pharmacokinetics of carboplatin. Clinical Pharmacokinetics 21(4):242-261.

Vega L, Ostrosky-Wegman P, Fortoul TI, Diaz C, Madrid V, Saavedra R.1999. Sodium arsenite reduces proliferation of human-activated T-cells by inhibition of the secretion of 
442

443

444

445

446

447

448

449

450

interleukin-2. Immunopharmacology and Immunotoxicology 21(2):203-20.

Wu MT, Lee LH, Ho CK, Liu CL, Wu TN, Wu SC, Lin LY, Cheng BH, Yang CY. 2003. Lifetime exposure to environmental tobacco smoke and cervical intraepithelial neoplasms among nonsmoking Taiwanese women. Archives of Environmental \& Occupational Health 58(6):353-359.

Wu S, Powers S, Zhu W, Hannun YA. 2016. Substantial contribution of extrinsic risk factors to cancer development. Nature 529(7584):43-7. DOI: 10.1038/nature16166.

Zitvogel L, Apetoh L, Ghiringhelli F, Kroemer G. 2008. Immunological aspects of cancer chemotherapy. Nature Reviews Immunology 8(1):59-73. 


\section{Table $\mathbf{1}$ (on next page)}

Demographic and clinical characteristics of women receiving cervical cancer treatment and participating in the study $(n=37)$ 
1

\begin{tabular}{|c|c|c|c|c|c|}
\hline Characteristics & Mean (n) & SD (\%) & Min. & $50^{\text {th } \% \text { tile }}$ & Max. \\
\hline Age (years) & 52.2 & 11.1 & 26 & 54 & 76 \\
\hline Body mass index $\left(\mathrm{kg} / \mathrm{m}^{2}\right)$ & 27.1 & 5.4 & 17 & 26.8 & 41.2 \\
\hline Smoker (yes) & $(9)$ & $(24.3)$ & - & - & - \\
\hline Tumor Stage & $(13)$ & $(35.2)$ & - & - & - \\
\hline IIB & $(14)$ & $(37.8)$ & - & - & - \\
\hline IIIA & $(10)$ & $(27)$ & - & - & - \\
\hline IIIB & 3.3 & 1.3 & 1 & 3 & 6 \\
\hline Change in tumor size (cm)
\end{tabular}

2 


\section{Table 2 (on next page)}

Urinary cotinine, arsenic, and phthalates metabolites measured in $\mathrm{n}=37$ women undergoing cervical cancer treatment and participating in the study ( $\mu \mathrm{g} / \mathrm{g}$ creatinine)

$\mathrm{LOD}=$ method limit of detection; $\mathrm{MBP}=$ mono butyl phthalate; $\mathrm{MBzP}=$ mono benzyl phthalate; MEHP = mono (2-ethylhexyl) phthalate; MEOHP = mono (2-ethyl-5-oxohexyl) phthalate; MEHHP = mono (2-ethyl-5-hydroxyhexyl) phthalate; \%MEHP = $100 \times(\mathrm{MEHP} /(\mathrm{MEHP}$ + MEOHP + MEHHP) on a molar basis 
1

\begin{tabular}{|c|c|c|c|c|c|c|}
\hline Analyte & Min. & $\mathbf{2 5}^{\text {th }}$ \%tile & $\mathbf{5 0}^{\text {th }} \%$ tile & $\mathbf{7 5}^{\text {th }} \%$ tile & Max. & $\begin{array}{c}\text { Geometric } \\
\text { mean }\end{array}$ \\
\hline Cotinine & $<$ LOD & $<$ LOD & 9.3 & 35.3 & 395.4 & 13.9 \\
\hline Arsenic & $<$ LOD & 7.4 & 13.2 & 20.8 & 115.4 & 13.1 \\
\hline MBP & $<$ LOD & $<$ LOD & 9.8 & 22 & 295.5 & 8.8 \\
\hline MBzP & $<$ LOD & 1.8 & 5.4 & 10.7 & 182.9 & 5 \\
\hline MEHP & 1.5 & 10.6 & 15.5 & 28.3 & 91.9 & 15.8 \\
\hline MEOHP & $<$ LOD & $<$ LOD & 2.8 & 8.2 & 34.1 & 3.5 \\
\hline MEHHP & $<$ LOD & 4.6 & 8.8 & 13.8 & 88.6 & 8.7 \\
\hline \%MEHP & 10.7 & 63.6 & 85.4 & 91.6 & 97.2 & 68.2 \\
\hline
\end{tabular}

2

3 


\section{Table 3 (on next page)}

Associations for urinary cotinine, arsenic, and phthalates $(\mu \mathrm{g} / \mathrm{l})$ with cervical cancer therapy response, adjusted for covariates using multiple linear regression models ${ }^{a}$

${ }^{\text {a }}$ Adjusted for baseline tumor size $(\mathrm{cm})$, age (years), urinary creatinine $(\mathrm{mg} / \mathrm{l})$, and urinary cotinine for arsenic and phthalates ( $\mu \mathrm{g} / \mathrm{l}) ;{ }^{b} \% \mathrm{MEHP}=100 \times(\mathrm{MEHP} /(\mathrm{MEHP}+\mathrm{MEOHP}+\mathrm{MEHHP}))$ on a molar basis.

$\mathrm{MBP}=$ mono butyl phthalate; $\mathrm{MBzP}=$ mono benzyl phthalate; $\mathrm{MEHP}=$ mono (2-ethylhexyl) phthalate; MEOHP = mono (2-ethyl-5-oxohexyl) phthalate; MEHHP = mono (2-ethyl-5-hydroxyhexyl) phthalate. 
1

\begin{tabular}{|c|c|c|c|c|c|}
\hline Predictors & $\mathbf{n}$ & $\boldsymbol{\beta}$ & \multicolumn{2}{|c|}{ 95\% CI } & P-value \\
\hline Cotinine & 37 & -0.001 & -0.005 & 0.003 & 0.590 \\
\hline Arsenic & 37 & 0.005 & -0.002 & 0.013 & 0.173 \\
\hline MBP & 35 & 0.0003 & -0.001 & 0.002 & 0.752 \\
\hline MBzP & 35 & -0.001 & -0.013 & 0.010 & 0.831 \\
\hline MEHP & 35 & 0.0007 & -0.006 & 0.007 & 0.817 \\
\hline MEOHP & 35 & -0.016 & -0.044 & 0.012 & 0.256 \\
\hline MEHHP & 35 & 0.0006 & -0.006 & 0.007 & 0.855 \\
\hline \%MEHP b $^{\text {35 }}$ & $\mathbf{0 . 0 1 5}$ & $\mathbf{0 . 0 0 3}$ & $\mathbf{0 . 0 3}$ & $\mathbf{0 . 0 1 9}$ \\
\hline
\end{tabular}

2

3 
Figure 1 (on next page)

Association between \%MEHP and response to cervical cancer treatment ${ }^{\mathrm{a}}$

${ }^{a}$ Adjusted for initial size of the tumor, urine cotinine, creatinine, and age \%MEHP $=100 \mathrm{x}$ (MEHP/(MEHP + MEOHP + MEHHP)) on a molar basis 


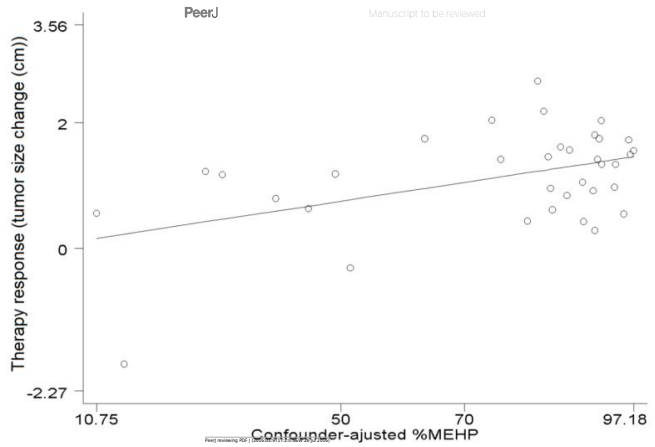

